The Journal of Nonlinear $\mathbf{S}_{\text {ciences and }}$ Applications

http://www.tjnsa.com

\title{
CONTROLLABILITY OF NONLOCAL IMPULSIVE FUNCTIONAL INTEGRODIFFERENTIAL EVOLUTION SYSTEMS
}

\author{
B. RADHAKRISHNAN ${ }^{1}$, K. BALACHANDRAN ${ }^{2}$
}

\begin{abstract}
In this paper, we establish a set of sufficient conditions for the controllability of nonlocal impulsive functional integrodifferential evolution systems with finite delay. The controllability results are obtained with out assuming the compactness condition on the evolution operator by using the semigroup theory and applying the fixed point approach. An example is provided to illustrate the theory.
\end{abstract}

\section{INTRODUCTION}

In various fields of engineering and physics, many problems that are related to linear viscoelasticity, nonlinear elasticity have mathematical models and are described by the problems of differential or integral equations or integrodifferential equations. Our work centers around the problems described by the integrodifferential models. The notion of controllability is of great importance in mathematical control theory. Many fundamental problems of control theory such as pole-assignment, stabilizability and optimal control may be solved under the assumption that the system is controllable. It means that it is possible to steer any initial state of the system to any final state in some finite time using an admissible control. As far as the controllability problems associated with finite-dimensional systems modelled by ODEs are concerned, this theory has evolved tremendously in the last decades to deal with nonlinearity and uncertainty of the physical models. In the finite-dimensional context, a system is controllable if and only if the algebraic Kalman rank condition is satisfied. According

Date: Received: May 21, 2011; Revised: November 30, 2011.

${ }^{*}$ Corresponding author

(c) 2011 N.A.G.

2000 Mathematics Subject Classification. 93B05; 34B10; 34G25.

Key words and phrases. Controllability; impulsive integrodifferential system; evolution operator; fixed point theorem; nonlocal condition. 
to this property, when a system is controllable for some time it is controllable for all time. But this is no longer true in the context of infinite-dimensional systems modelled by PDEs.

The controllability of nonlinear systems in finite-dimensional space by means of fixed point principles [1]. This concept has been extended to infinite-dimensional spaces by applying semigroup theory ([19]). Controllability of nonlinear systems with different types of nonlinearity has been studied by many authors with the help of fixed point principles ([2]). Many systems in physics and biology exhibit impulsive dynamical behavior due to sudden jumps at certain instants in the evolution process. Differential equations involving impulsive effects occur in many applications: radiation of electromagnetic waves, population dynamics, biological systems, the abrupt increase of glycerol in fed-batch culture etc.,([12]). For the basic theory of impulsive differential equations can refer to $([15],[21])$ and Fu et al. ([11]) studied the existence for neutral impulsive differential inclusions with nonlocal conditions.

Controllability of linear and nonlinear impulsive systems in finite dimensional space has been discussed by many authors ([13],[14],[22]). Li et al. ([16]) and Chang ([9]) extended the results to impulsive functional differential systems in Banach spaces by using compact semigroup and the Schaefer fixed point theorem. Balchandran and Park ([3]) studied the existence and uniqueness of a mild solution for a functional integrodifferential equation with nonlocal conditions. Byszewski and Acka ([7]) established the existence and uniqueness and continuous dependence of mild solution of semilinear functional differential equation with nonlocal condition of the form

$$
\begin{array}{r}
\frac{d u(t)}{d t}+A u(t)=f\left(t, u_{t}\right), \quad t \in[0, a], \\
u(s)+\left[g\left(u_{t_{1}}, \ldots, u_{t_{p}}\right)\right](s)=\varphi(s), \quad s \in[-r, 0]
\end{array}
$$

where $0<t_{1}<\ldots<t_{p} \leq a,-A$ is the infinitesimal generator of a $C_{0}-$ semigroup of operators on a Banach space. Theorems about the existence, uniqueness and stability of solutions of functional differential equations abstract evolution Cauchy problems were studied by Byszewski and Lakshmikantham ([5]), by Byszewski ([6],[7],[8]), by Balachandran and Chandrasekaran ([4]), and by Lin and Liu ([17]). In this paper we study the controllability of nonlocal impulsive functional integrodifferential with evolution systems and by using the results of $([3],[7],[8],[19])$.

\section{Preliminaries}

Consider the following nonlocal impulsive nonlinear integrodifferential evolution system

$$
\begin{aligned}
x^{\prime}(t) & =A(t) x(t)+B u(t)+f\left(t, x_{t}, \int_{0}^{t} h\left(t, s, x_{s}\right) d s\right), t \neq t_{i}, t \in J, \\
x(s) & +\left[g\left(x_{t_{1}}, \ldots, x_{t_{p}}\right)\right](s)=\varphi(s), s \in[-r, 0], \\
\left.\Delta x\right|_{t=t_{i}} & =I_{i}\left(x\left(t_{i}^{-}\right)\right), \quad i=1,2, \ldots, m,
\end{aligned}
$$

where the state variable $x(\cdot)$ takes values in a Banach space $X$ and the control function $u(\cdot)$ is given in $\mathcal{L}^{2}(J, V)$, a Banach space of admissible control functions with $V$ as a Banach space and $J=[0, b], \Omega=\{(t, s): 0 \leq s \leq t \leq b\}$. Here $A(t)$ is a closed linear densely defined operator in $X$ and $\mathrm{B}$ is a bounded linear operator from $V$ into $X$. Further $f: J \times X \times X \rightarrow X, h: \Omega \times X \rightarrow X, I_{i}: X \rightarrow X,\left.\Delta x\right|_{t=t_{i}}=x\left(t_{i}^{+}\right)-x\left(t_{i}^{-}\right)$, for 
all $i=1,2, \ldots, m ; 0=t_{0}<t_{1}<t_{2}<\ldots<t_{m}<t_{m+1}=b$; and the nonlocal function $g:[\mathcal{P C}([-r, 0], X)]^{p} \rightarrow X$ are given functions. The history $x_{t}$ represents the function $x_{t}:(-r, 0] \rightarrow X$ defined by $x_{t}(\theta)=x(t+\theta)$, for $t \in[0, b], \theta \in[-r, 0]$.

Denote $J_{0}=\left[0, t_{1}\right], J_{i}=\left(t_{i}, t_{i+1}\right], i=1,2, \ldots, m$ and define the following space:

Let $\mathcal{P C}([-r, b], X)=\{x: x$ is a function from $[-r, b]$ into $X$ such that $x(t)$ is continuous at $t \neq t_{i}$ and left continuous at $t=t_{i}$ and the right limit $x\left(t_{i}^{+}\right)$exists for $\left.i=1,2, \ldots, m\right\}$. Similarly as in $([12],[18])$, we see that $\mathcal{P C}([-r, b], X)$ is a Banach space with norm

$$
\|x\|_{\mathcal{P C}}=\sup _{t \in[0, b]}\|x(t)\|
$$

For the family $\{A(t): 0 \leq t \leq b\}$ of linear operators, we assume the following hypotheses:

(A1) $A(t)$ is a closed linear operator and the domain $\mathcal{D}(A)$ of $\{A(t): 0 \leq t \leq b\}$ is dense in the Banach space $X$ and independent of $t$.

(A2) For each $t \in[0, b]$, the resolvent $R(\lambda, A(t))=(\lambda I-A(t))^{-1}$ of $A(t)$ exists for all $\lambda$ with $R e \lambda \leq 0$ and $\|R(\lambda, A(t))\| \leq C(|\lambda|+1)^{-1}$.

(A3) For any $t, s, \tau \in[0, b]$, there exists a $0<\delta<1$ and $L>0$ so that

$$
\left\|(A(t)-A(\tau)) A^{-1}(s)\right\| \leq L|t-\tau|^{\delta} .
$$

Statements $(A 1)-(A 3)$ implies that there exists a family of evolution operator $U(t, s)$ (see [10]).

The family $\{A(t): 0 \leq t \leq b\}$ generates a unique linear evolution system $\{U(t, s): 0 \leq$ $s \leq t \leq b\}$ satisfying the following properties:

(a) $U(t, s) \in L(X)$ the space of bounded linear transformation on $X$, whenever $0 \leq s \leq$ $t \leq b$ and for each $x \in X$, the mapping $(t, s) \rightarrow U(t, s) x$ is continuous.

(b) $U(t, s) U(s, \tau)=U(t, \tau)$ for $0 \leq \tau \leq s \leq t \leq b$.

(c) $U(t, t)=I$.

Definition 2.1. A solution $x(\cdot) \in \mathcal{P C}([-r, b], X)$ is said to be a mild solution of $(2.1)-$ (2.3) if $x(s)+\left[g\left(x_{t_{1}}, \ldots, x_{t_{p}}\right)\right](s)=\varphi(s), s \in[-r, 0],\left.\Delta x\right|_{t=t_{i}}=I_{i}\left(x\left(t_{i}^{-}\right)\right), i=1,2, \ldots, m$; the restriction of $x(\cdot)$ to the interval $J_{i}(i=0,1, \ldots, m)$ is continuous and the following 
conditions are satisfied:

$$
\begin{aligned}
(i) x(t)= & U(t, 0) \varphi(0)-U(t, 0)\left[g\left(x_{t_{1}}, \ldots, x_{t_{p}}\right)\right](0)+\int_{0}^{t} U(t, s)[B u(s) \\
& \left.+f\left(s, x_{s}, \int_{0}^{s} h\left(s, \tau, x_{\tau}\right) d \tau\right)\right] d s+\sum_{0<t_{i}<t} U\left(t, t_{i}\right) I_{i}\left(x\left(t_{i}^{-}\right)\right), \quad t \in[0, b], \\
(\text { ii }) x(s)+ & {\left[g\left(x_{t_{1}}, \ldots, x_{t_{p}}\right)\right](s)=\varphi(s), \quad s \in[-r, 0) . }
\end{aligned}
$$

To study the controllability problem we assume the following hypotheses:

(H1) $A(t)$ generates a family of evolution operators $U(t, s)$ in $X$ and there exists a constant $M>0$ such that

$$
\|U(t, s)\| \leq M, \text { for } 0 \leq s \leq t \leq b .
$$

(H2) The linear operator $W: \mathcal{L}^{2}(J, V) \rightarrow X$ defined by

$$
W u=\int_{0}^{b} U(b, s) B u(s) d s
$$

has an inverse operator $W^{-1}$, which takes values in $\mathcal{L}^{2}(J, V) / \operatorname{ker} W$ and there exists a positive constant $K$ such that $\left\|B W^{-1}\right\| \leq K$.

(H3) The nonlinear function $f: J \times X \times X \rightarrow X$ is continuous and there exist constants $L_{B}>0, L_{0}>0$ such that

$$
\begin{aligned}
\left\|f\left(t, x_{t}, u_{t}\right)-f\left(t, y_{t}, v_{t}\right)\right\| \leq & L_{B}(\|x-y\|+\|u-v\|), \text { for } x, y, u, v \in X, t \in J, \\
& L_{0}=\max _{t \in J}\|f(t, 0,0)\| .
\end{aligned}
$$

(H4) For each $(t, s) \in \Omega$, the function $h: \Omega \times X \rightarrow X$ is continuous and there exist constants $N_{B}>0$ and $N_{0}>0$ such that

$$
\begin{gathered}
\int_{0}^{t}\left\|h\left(t, s, x_{s}\right)-h\left(t, s, y_{s}\right)\right\| d s \leq N_{B}\|x-y\|, \text { for } x, y \in X, t, s \in J, \\
N_{0}=\max \left\{\int_{0}^{t}\|h(t, s, 0)\| d s: t, s \in \Omega\right\} .
\end{gathered}
$$

(H5) $I_{i}: X \rightarrow X$ is continuous and there exist constants $l_{i}$ such that

$$
\left\|I_{i}(x)-I_{i}(y)\right\| \leq l_{i}\|x-y\|, \quad i=1,2, \ldots, m,
$$

for each $x, y \in X$.

(H6) $g:[\mathcal{P C}([-r, 0], X)]^{p} \rightarrow X$ is continuous and there exists a constant $G_{B}>0$ such that

$$
\|\left[g\left(x_{t_{1}}, \ldots, x_{t_{p}}\right](s)-\left[g\left(y_{t_{1}}, \ldots, y_{t_{p}}\right](s)\left\|\leq G_{B}\right\| x-y \|_{\mathcal{P C}},\right.\right.
$$

for each $x, y \in \mathcal{P C}([-r, b], X), s \in[-r, 0]$,

$$
G_{0}=\max \left\{\|\left[g\left(x_{t_{1}}, \ldots, x_{t_{p}}\right](s) \|: x, y \in \mathcal{P C}([-r, b], X), s \in[-r, 0]\right\} .\right.
$$


(H7) There exist a positive constant $\rho>0$ such that

$$
M(1+b M K)\left[\|\varphi(0)\|+G_{0}+b\left\{L_{B}\left[N_{0}+\left(1+N_{B}\right) r\right]+L_{0}\right\}+\sum_{i=1}^{m} l_{i}\right]+b M K\left\|x_{1}\right\| \leq \rho .
$$

Moreover, let us put $\lambda=\sum_{i=1}^{m} l_{i}$ and

$$
\gamma=M(1+b M K)\left[G_{B}+b L_{B}+b N_{B}+\lambda\right] .
$$

Definition 2.2. [20] The system (2.1) - (2.3) is said to be controllable on the interval $J$ if for every $x_{1} \in X$ and $\left[g\left(x_{t_{1}}, \ldots, x_{t_{p}}\right](s) \in \mathcal{P C}([-r, b], X)\right.$, there exists a control $u \in \mathcal{L}^{2}(J, V)$ such that the mild solution $x(t)$ of $(1)-(3)$ satisfies $x(0)=x_{0}$ and $x(b)=x_{1}$.

\section{Controllability Result}

Theorem 3.1. If the conditions $(H 1)-(H 7)$ are satisfied and if $0 \leq \gamma<1$, then the system $(2.1)-(2.3)$ is controllable on $J$.

Proof. Introduce the operator $\Gamma$ on the Banach space $\mathcal{P C}([-r, b], X)$ by the formula:

$$
(\Gamma x)(t)=\left\{\begin{array}{l}
\varphi(t)-\left[g\left(x_{t_{1}}, \ldots, x_{t_{p}}\right)\right](t), \quad t \in[-r, 0], \\
U(t, 0) \varphi(0)-U(t, 0)\left[g\left(x_{t_{1}}, \ldots, x_{t_{p}}\right)\right](0)+\int_{0}^{t} U(t, s) B u(s) d s \\
+\int_{0}^{t} U(t, s) f\left(s, x_{s}, \int_{0}^{s} h\left(s, \tau, x_{\tau}\right) d \tau\right) d s+\sum_{0<t_{i}<t} U\left(t, t_{i}\right) I_{i}\left(x\left(t_{i}^{-}\right)\right), \quad t \in J .
\end{array}\right.
$$

Using hypothesis $(H 2)$ for an arbitrary function $x(\cdot)$, define the control

$$
\begin{aligned}
u(t)= & W^{-1}\left[x_{1}-U(b, 0)\left[\varphi(0)-\left(g\left(x_{t_{1}}, \ldots, x_{t_{p}}\right)\right)(0)\right]\right. \\
& \left.-\int_{0}^{b} U(b, s) f\left(s, x_{s}, \int_{0}^{s} h\left(s, \tau, x_{\tau}\right) d \tau\right) d s-\sum_{0<t_{i}<b} U\left(b, t_{i}\right) I_{i}\left(x\left(t_{i}^{-}\right)\right)\right](t) .
\end{aligned}
$$

We shall now show that when using this control, the operator

$$
\Gamma: \mathcal{P C}([-r, b], X) \rightarrow \mathcal{P C}([-r, b], X)
$$


defined by

$$
\begin{aligned}
(\Gamma x)(t)= & U(t, 0)\left[\varphi(0)-\left[g\left(x_{t_{1}}, \ldots, x_{t_{p}}\right)\right](0)\right]+\int_{0}^{t} U(t, s) B W^{-1}\left[x_{1}-U(b, 0) \varphi(0)\right. \\
& \left.-U(b, 0)\left[g\left(x_{t_{1}}, \ldots, x_{t_{p}}\right)\right](0)\right]-\int_{0}^{b} U(b, s) f\left(s, x_{s}, \int_{0}^{s} h\left(s, \tau, x_{\tau}\right) d \tau\right) d s \\
& \left.-\sum_{0<t_{i}<b} U\left(b, t_{i}\right) I_{i}\left(x\left(t_{i}^{-}\right)\right)\right](s) d s+\int_{0}^{t} U(t, s) f\left(s, x_{s}, \int_{0}^{s} h\left(s, \tau, x_{\tau}\right) d \tau\right) d s \\
& +\sum_{0<t_{i}<t} U\left(t, t_{i}\right) I_{i}\left(x\left(t_{i}^{-}\right)\right)
\end{aligned}
$$

has a fixed point $x(\cdot)$. To prove the controllability, it is enough to show that the operator $\Gamma$ has a fixed point in $\mathcal{P C}([-r, b], X)$ and since all the functions involved in the operator are continuous and therefore $\Gamma$ is continuous.

Let $\mathcal{S}$ be a nonempty closed subset of $\mathcal{P C}([-r, b], X)$ defined by

$$
\mathcal{S}=\left\{x: x \in \mathcal{P C}([-r, b], X),\|x(t)\|_{\mathcal{P C}} \leq r, 0 \leq t \leq b\right\}
$$

First we show that $\Gamma$ maps $\mathcal{S}$ into $\mathcal{S}$. For $x \in \mathcal{S}$, we have

$$
\begin{aligned}
\|(\Gamma x)(t)\| \leq \| & U(t, 0)\left[\varphi(0)-\left[g\left(x_{t_{1}}, \ldots, x_{t_{p}}\right)\right](0)\right]\|+\| \int_{0}^{t} U(t, s) B W^{-1}\left[x_{1}-U(b, 0) \varphi(0)\right. \\
& \left.-U(b, 0)\left[g\left(x_{t_{1}}, \ldots, x_{t_{p}}\right)\right](0)\right]-\int_{0}^{b} U(b, s) f\left(s, x_{s}, \int_{0}^{s} h\left(s, \tau, x_{\tau}\right) d \tau\right) d s \\
& \left.-\sum_{0<t_{i}<b} U\left(b, t_{i}\right) I_{i}\left(x\left(t_{i}^{-}\right)\right)\right](s) d s\|+\| \int_{0}^{t} U(t, s) f\left(s, x_{s}, \int_{0}^{s} h\left(s, \tau, x_{\tau}\right) d \tau\right) d s \| \\
& +\left\|\sum_{0<t_{i}<t} U\left(t, t_{i}\right) I_{i}\left(x\left(t_{i}^{-}\right)\right)\right\| \\
\leq & M\|\varphi(0)\|+M G_{0}+M K \int_{0}^{t}\left[\left\|x_{1}\right\|+M\|\varphi(0)\|+M G_{0}\right. \\
& \left.+M \int_{0}^{b}\left\|f\left(s, x_{s}, \int_{0}^{s} h\left(s, \tau, x_{\tau}\right) d \tau\right)\right\| d s+M \sum_{0<t_{i}<b} l_{i}\right] d s \\
& +M \int_{0}^{b}\left\|f\left(s, x_{s}, \int_{0}^{s} h\left(s, \tau, x_{\tau}\right) d \tau\right)\right\| d s+M \sum_{0<t_{i}<t} l_{i}
\end{aligned}
$$


Since from assumptions $(H 3)$ and $(H 4)$, we have

$$
\begin{aligned}
\left\|f\left(s, x_{s}, \int_{0}^{s} h\left(s, \tau, x_{\tau}\right) d \tau\right)\right\| & \leq\left\|f\left(s, x_{s}, \int_{0}^{s} h\left(s, \tau, x_{\tau}\right) d \tau\right)-f(s, 0,0)+f(s, 0,0)\right\| \\
& \leq\left\|f\left(s, x_{s}, \int_{0}^{s} h\left(s, \tau, x_{\tau}\right) d \tau\right)-f(s, 0,0)\right\|+\|f(s, 0,0)\| \\
& \left.\leq L_{B}\left[\left\|x_{s}\right\|+\| \int_{0}^{s} h\left(s, \tau, x_{\tau}\right) d \tau\right) \|\right]+L_{0} \\
& \leq L_{B}\left[\left\|x_{s}\right\|+\int_{0}^{s}\left\|h\left(s, \tau, x_{\tau}\right)-h(s, \tau, 0)\right\| d \tau+\int_{0}^{s}\|h(s, \tau, 0)\| d \tau\right]+L_{0} \\
& \leq L_{B}\left[\left\|x_{s}\right\|+N_{B}\left\|x_{s}\right\|+N_{0}\right]+L_{0} \\
& \leq L_{B}\left[N_{0}+\left(1+N_{B}\right)\left\|x_{s}\right\|\right]+L_{0}
\end{aligned}
$$

there holds

$$
\begin{aligned}
\|(\Gamma x)(t)\| \leq & M\|\varphi(0)\|+M G_{0}+b M K\left[\left\|x_{1}\right\|+M\|\varphi(0)\|+M G_{0}\right. \\
& \left.+b M\left\{L_{B}\left[N_{0}+\left(1+N_{B}\right)\left\|x_{s}\right\|\right]+L_{0}\right\}+M \sum_{k=1}^{m} l_{i}\right] \\
& +b M\left\{L_{B}\left[N_{0}+\left(1+N_{B}\right)\left\|x_{s}\right\|\right]+L_{0}\right\}+M \sum_{0<t_{i}<t} l_{i} \\
\leq & M(1+b M K)\left[\|\varphi(0)\|+G_{0}+b\left\{L_{B}\left[N_{0}+\left(1+N_{B}\right) r\right]+L_{0}\right\}+\sum_{i=1}^{m} l_{i}\right]+b M K\left\|x_{1}\right\| .
\end{aligned}
$$

From $(H 7)$, one gets $\|(\Gamma x)(t)\| \leq \rho$, therefore $\Gamma$ maps $\mathcal{S}$ into itself.

Now we shall show that $\Gamma$ is a contraction on $\mathcal{S}$. For this purpose consider two differences as follows:

$$
\begin{array}{r}
(\Gamma x)(t)-(\Gamma y)(t)=\left[g\left(x_{t_{1}}, \ldots, x_{t_{p}}\right)\right](t)-\left[g\left(y_{t_{1}}, \ldots, y_{t_{p}}\right)\right](t), \\
\text { for } x, y \in \mathcal{P C}([-r, b], X), t \in[-r, 0),
\end{array}
$$


and

$$
\begin{aligned}
& (\Gamma x)(t)-(\Gamma y)(t) \\
& =U(t, 0)\left[\left(g\left(x_{t_{1}}, \ldots, x_{t_{p}}\right)\right)(0)-\left(g\left(y_{t_{1}}, \ldots, y_{t_{p}}\right)\right)(0)\right]+\int_{0}^{t} U(t, \eta) B W^{-1} \\
& \times\left\{U(b, 0)\left[\left(g\left(x_{t_{1}}, \ldots, x_{t_{p}}\right)\right)(0)-\left(g\left(y_{t_{1}}, \ldots, y_{t_{p}}\right)\right)(0)\right]\right. \\
& +\int_{0}^{b} U(b, s)\left[f\left(s, x_{s}, \int_{0}^{s} h\left(s, \tau, x_{\tau}\right) d \tau\right)-f\left(s, y_{s}, \int_{0}^{s} h\left(s, \tau, y_{\tau}\right) d \tau\right)\right] d s \\
& \left.+\sum_{0<t_{i}<b} U\left(b, t_{i}\right)\left[I_{i}\left(x\left(t_{i}^{-}\right)\right)-I_{k}\left(y\left(t_{i}^{-}\right)\right)\right]\right\}(\eta) d \eta \\
& +\int_{0}^{t} U(t, s)\left[f\left(s, x_{s}, \int_{0}^{s} h\left(s, \tau, x_{\tau}\right) d \tau\right)-f\left(s, y_{s}, \int_{0}^{s} h\left(s, \tau, y_{\tau}\right) d \tau\right)\right] d s \\
& +\sum_{0<t_{i}<t} U\left(t, t_{i}\right)\left[I_{i}\left(x\left(t_{i}^{-}\right)\right)-I_{i}\left(y\left(t_{i}^{-}\right)\right)\right], \quad x, y \in \mathcal{P C}([-r, b], X), \quad t \in J .
\end{aligned}
$$

From (3.1) and (H6), we have

$$
\|(\Gamma x)(t)-(\Gamma y)(t)\| \leq G_{B}\|x-y\|_{\mathcal{P C}}, \quad \text { for } \quad x, y \in \mathcal{P C}([-r, b], X), t \in[-r, 0)
$$

Moreover, by (3.2), (H3) - (H6), we obtain

$$
\begin{aligned}
\|(\Gamma x)(t)-(\Gamma y)(t)\| \\
\leq\left\|U(t, 0)\left[\left(g\left(x_{t_{1}}, \ldots, x_{t_{p}}\right)\right)(0)-\left(g\left(y_{t_{1}}, \ldots, y_{t_{p}}\right)\right)(0)\right]\right\|+\| \int_{0}^{t} U(t, \eta) B W^{-1} \\
\times\left\{U(b, 0)\left[\left(g\left(x_{t_{1}}, \ldots, x_{t_{p}}\right)\right)(0)-\left(g\left(y_{t_{1}}, \ldots, y_{t_{p}}\right)\right)(0)\right]\right. \\
+\int_{0}^{b} U(b, s)\left[f\left(s, x_{s}, \int_{0}^{s} h\left(s, \tau, x_{\tau}\right) d \tau\right)-f\left(s, y_{s}, \int_{0}^{s} h\left(s, \tau, y_{\tau}\right) d \tau\right)\right] d s \\
\left.+\sum_{0<t_{i}<b} U\left(b, t_{i}\right)\left[I_{i}\left(x\left(t_{i}^{-}\right)\right)-I_{i}\left(y\left(t_{i}^{-}\right)\right)\right]\right\}(\eta) d \eta \| \\
+\left\|\int_{0}^{t} U(t, s)\left[f\left(s, x_{s}, \int_{0}^{s} h\left(s, \tau, x_{\tau}\right) d \tau\right)-f\left(s, y_{s}, \int_{0}^{s} h\left(s, \tau, y_{\tau}\right) d \tau\right)\right] d s\right\| \\
+\left\|\sum_{0<t_{i}<t} U\left(t, t_{i}\right)\left[I_{i}\left(x\left(t_{i}^{-}\right)\right)-I_{i}\left(y\left(t_{i}^{-}\right)\right)\right]\right\|
\end{aligned}
$$




$$
\begin{aligned}
\leq & M G_{B}\|x-y\|_{\mathcal{P C}}+M K \int_{0}^{t}\left[M G_{B}\|x-y\|_{\mathcal{P C}}\right. \\
& \left.+M \int_{0}^{b} L_{B}\left[\|x-y\|+N_{B}\|x-y\|\right] d s+M \sum_{i=1}^{m} l_{i}\|x-y\|_{\mathcal{P C}}\right] d \eta \\
& +M \int_{0}^{b} L_{B}\left[\|x-y\|+N_{B}\|x-y\|\right] d s+M \sum_{0<t_{i}<t} l_{i}\|x-y\|_{\mathcal{P C}} \\
\leq & M G_{B}\|x-y\|_{\mathcal{P C}}+b M K\left[M G_{B}\|x-y\|_{\mathcal{P C}}+b M L_{B}\|x-y\|+b M N_{B}\|x-y\|\right. \\
& \left.+M \lambda\|x-y\|_{\mathcal{P C}}\right]+b M L_{B}\|x-y\|+b M N_{B}\|x-y\|+M \lambda\|x-y\|_{\mathcal{P C}} \\
\leq & M(1+b M K)\left[G_{B}+b L_{B}+b N_{B}+\lambda\right]\|x-y\|_{\mathcal{P C} .}
\end{aligned}
$$

From (3.3) and (3.4), we get

$$
\|(\Gamma x)(t)-(\Gamma y)(t)\| \leq \gamma\|x-y\|_{\mathcal{P C}}, \text { for } x, y \in \mathcal{P C}([-r, b], X),
$$

where $\gamma=M(1+b M K)\left[G_{B}+b L_{B}+b N_{B}+\lambda\right]$.

Since $\gamma<1$, then (3.5) shows that the operator $\Gamma$ is a contraction on $\mathcal{P C}([-r, b], X)$. Also, $\Gamma$ satisfies the Banach contraction theorem. Hence there exists a unique fixed point $x \in \mathcal{P C}([-r, b], X)$ such that $(\Gamma x)(t)=x(t)$ and this point is the mild solution of the system $(2.1)-(2.3)$ and $(\Gamma x)(b)=x(b)=x_{1}$, which implies that the given system is controllable.

\section{EXAMPLE}

Consider the following partial integrodifferential equation with impulses:

$$
\begin{aligned}
\frac{\partial}{\partial t} z(t, y)= & \frac{\partial^{2}}{\partial y^{2}} z(t, y)+a(t, y) z(t, y)+\mu(t, y) \\
& +\eta_{1}(y) \int_{-r}^{t} \sin z_{t}(s, y) d s+\eta_{2} \int_{-r}^{t} \int_{0}^{s} e^{-z_{t}(\tau, y)} d \tau d s, \\
0 \leq y \leq 1, \quad t \in J=[0, b], & t \geq 0, \\
z(t, 0)= & z(t, 1)=0, \quad \quad \quad \sum_{i=1}^{m} e_{i} \varphi_{t_{i}}(s, y)=z_{0}(y), \quad \varphi \in \mathcal{P C}, \quad 0 \leq y \leq 1, \\
z(0, y)+ & I_{i}(z(y))=\left(\beta_{i}|z(y)|+t_{i}\right)^{-1}, \quad z \in X, 1 \leq i \leq m,
\end{aligned}
$$

where $a(t, y)$ continuous on $0 \leq y \leq 1,0 \leq t \leq b$ and constants $e_{i}, \beta_{i}$ are small and $\eta_{1}(y)$ is continuous on $[0,1], \eta_{2}>0$.

Let us take $X=V=\mathcal{L}^{2}[0,1]$ be endowed with the usual norm $\left.|\cdot|\right|_{\mathcal{L}^{2}}$.

Put $x_{t}(s)=z_{t}(s, \cdot)$ and $u(t)=\mu(t, \cdot)$ where $\mu: J \times[0,1] \rightarrow[0,1]$ be the control function is continuous. 
Define the operators $g, f$ by

$$
g\left(\varphi_{t_{i}}(s, \cdot)\right)(s)=\sum_{i=1}^{m} e_{i} \varphi_{t_{i}}(s, y)
$$

and

$$
f\left(t, x_{t}, \mathcal{H}\left(x_{t}\right)\right)=\eta_{1}(\cdot) \int_{-r}^{t} \sin z_{t}(s, y) d s+\mathcal{H}\left(x_{t}\right)
$$

where

$$
\mathcal{H}\left(x_{t}\right)=\eta_{2} \int_{-r}^{t} \int_{0}^{s} e^{-z_{t}(\tau, y)} d \tau d s .
$$

Let $A: \mathcal{D}(A) \subset X \rightarrow X$ be the operator defined by $A z=z^{\prime \prime}$ with the domain $D(A)=\{z \in$ $X: z, z^{\prime}$ are absolutely continuous, $\left.z^{\prime \prime} \in X, z(0)=z(\pi)=0\right\}$. It is well known that $A$ is the infinitesimal generator of a $C_{0}-\operatorname{semigroup~}(T(t))_{t>0}$ on $X$. Furthermore $A$ has a discrete spectrum with eigenvalues $-n^{2}, n \in N$ and corresponding normalized eigenfunctions given by $z_{n}(y)=\sqrt{2 / \pi} \sin n y, n=1,2,3, \ldots$.

In addition, $\left\{z_{n}: n \in N\right\}$ is an orthonormal basis of $X$ and

$$
T(t) z=\sum_{n=1}^{\infty} e^{-n^{2} t}\left(x, z_{n}\right) z_{n}
$$

for $x \in X$ and $t \geq 0$.

Now we define the operator $A(t) z=A z(y)+a(t, y) z, z \in \mathcal{D}(A(t)), t \geq 0, y \in[0, \pi]$, where $\mathcal{D}(A(t))=\mathcal{D}(A), t \geq 0$. By assuming that $y \rightarrow a(t, y)$ is continuous in $t$, and there exists $\rho>0$ such that $a(t, y) \leq-\rho$, for all $t \in J, y \in[0, \pi]$, it follows that the system

$$
\begin{aligned}
& z^{\prime}(t)=A(t) z(t) \quad t \geq s, \\
& z(0)=z_{0}
\end{aligned}
$$

generates an evolution system $U(t, s)$ satisfying assumptions $(A 1)-(A 3)$. Furthermore, $U(t, s) z=T(t-s) \exp \left(\int_{s}^{t} a(\tau, y) d \tau\right) z$ for $z \in X$ and $\| U\left(t, s \| \leq e^{-(1+\sigma)(t-s)}\right.$ for every $t \geq s$.

With this choice $A(t), I_{i}, f, g$ and $B=I$ the identity operator, we see that equation $(4.1)-(4.4)$ can be written in the abstract formulation of (2.1) - (2.3). Assume that the linear operator $W$ from $\mathcal{L}^{2}(J, V) / \operatorname{ker} W$ into $X$ is defined by

$$
W u=\int_{0}^{b} T(b-s) e^{\int_{s}^{b} a(\tau, y) d \tau} \mu(s, \cdot) d s,
$$

has an invertible operator and satisfies the condition $(H 2)$.

Further the conditions $(H 3)-(H 6)$ are satisfied and it is possible to choose $e_{i}, \beta_{i}, \eta_{1}, \eta_{2}$ in such a way that the constant $\gamma<1$. Hence by Theorem 3.1. the system $(4.1)-(4.4)$ is controllable on $J$. 


\section{REFERENCES}

[1] K.Balachandran and J.P.Dauer, Controllability of nonlinear systems via Fixed Point Theorems, J. Optim. Theory and Appl., 53 (1987), 345-352.

[2] K.Balachandran and J.P.Dauer, Controllability of nonlinear systems in Banach spaces; A survey, J. Optim. Theory and Appl., 115 (2002), 7-28.

[3] K.Balachandran and J.Y.Park, Existence of a mild solution of a functional integrodifferential equation with nonlocal condition, Bull.Korean Math. Soc., 38 (2001), 175-182.

[4] K.Balachandran and M.Chandrasekaran, Existence of solutions of a delay differential equation with nonlocal condition, Indian. J. Pure Appl. Math., 27 (1996), 443-449.

[5] L.Byszewski and V.Lakshmikantham, Theorem about the existence and uniqueness of a solution of a nonlocal abstract Cauchy problem in a banach space, Appl. Anal. 40(1991), 11-19.

[6] L.Byszewski, Theorems about the existence and uniqueness of solutions of a semilinear evolution nonlocal Cauchy problem, J. Math. Anal. Appl., 162(1991), 494-505.

[7] L.Byszewski and H.Akca, On a mild solutions of a semilinear functional differential evolution nonlocal problem, J. Appl. Math. Stoch. Anal., 3 (1997), 265-271.

[8] L.Byszewski and H.Akca, Existence of solutions of a semilinear functional-differential evolution nonlocal problem, Nonlinear Anal., 34(1998), 65-72.

[9] Y.K.Chang, Controllability of impulsive functional differential systems with infinite delay in Banach spaces, Chaos, Solitons and Fractals 33 (2007), 1601-1609.

[10] A.Friedman, Partial Differential Equations, Holt, Rinehart and Winston, New York, 1969.

[11] X.Fu and Y.Cao Existence for neutral impulsive differential inclusions with nonlocal conditions, Nonlinear Anal., 68(2008), 3707-3718.

[12] D.Guo, X.Liu, Extremal solutions of nonlinear impulsive integrodifferential equations in Banach spaces, J. Math. Anal. Appl., 177 (1993), 538-552.

[13] R.K.George, A.K.Nandakumaran, and A.Arapostathis, A note on controllability of impulsive systems, J. Math. Anal. Appl., 241(2000), 276-283.

[14] Z.H.Guan, T.H.Qian and X.Yu, On controllability and observability for a class of impulsive systems, Systems and Control Letters, 47(2002), 247-257.

[15] V.Lakshmikantham, D.D.Bainov and P.S.Simeonov, Theory of Impulsive Differential Equations, World Scientific, Singapore, 1989.

[16] M.Li, M.Wang and F.Zhang, Controllability of impulsive functional differential systems in Banach spaces, Chaos, Solitons and Fractals, 29 (2006), 175-181.

[17] Y.Lin and J.H.Liu, Semilinear integrodifferential equations with nonlocal Cauchy problem, Nonlinear Anal., 26 (1996), 1023-1033.

[18] J.Liang, J.H. Liu and T.J.Xiao, Nonlocal impulsive problems for nonlinear differential equations in Banach spaces, Math. Comp. Modelling, 49 (2009), 798-804.

[19] A.Pazy, Semigroups of Linear Operators and Applications to Partial Differential Equations, SpringerVerlag, New York, 1983.

[20] B.Radhakrishnan, K.Balachandran, Controllability of impulsive neutral functional evolution integrodifferential systems with infinite delay, Nonlinear Anal.: Hybrid Sys., 5 (2011), 655-670.

[21] A.M.Samoilenko and N.A.Perestyuk, Impulsive Differential Equations, World Scientific, Singapore, 1995.

[22] G.Xie and L.Wang, Controllability and observability of a class of linear impulsive systems, J. Math. Anal. Appl., 304 (2005), 336-355.

${ }^{1}$ Department of Mathematics, Bharathiar University, Coimbatore, Tamil nadu, India

2 Department of Mathematics, Bharathiar University, Coimbatore, Tamil nadu, India

E-mail address: radhakrishnanb1985@gmail. $\mathrm{com}^{1}$, kb.maths.bu@gmail. $\mathrm{com}^{2}$ 